\title{
Optic Nerve Hypoplasia, Corpus Callosum Agenesis, Cataract, and Lissencephaly in a Neonate with a Novel COL4A1 Mutation
}

\author{
Lisa Grego ${ }^{a}$ Silvia Pignatto ${ }^{a} \quad$ Nicolò Rassu $^{a} \quad$ Eva Passone $^{b}$ \\ Paola Cogo ${ }^{\text {b }}$ Paolo Lanzetta ${ }^{a}$ \\ a Department of Medicine - Ophthalmology, University of Udine, Udine, Italy; ${ }^{b}$ Department \\ of Medicine - Pediatrics, University of Udine, Udine, Italy
}

\section{Keywords}

COL4A 1 - Corpus callosum agenesis · Optic nerve hypoplasia $\cdot$ Cataract

\begin{abstract}
We report the case of a girl with a novel mutation of the COL4A gene (c.2716+2T>C) presenting microcephaly, parenchymal hemorrhages, lissencephaly, and bilateral cataracts, associated with agenesis of the corpus callosum and hypoplasia of the optic nerve. COL4A1, located on chromosome 13, encodes the $\alpha 1$ chain of type IV collagen, a key component of the basement membrane in various organs, such as eye, brain, kidneys, and muscles. Different mutations have been described and may remain asymptomatic or determine porencephaly, cerebral hemorrhages, renal cysts, hematuria, and dysgenesis of the anterior segment of the eye.
\end{abstract}

\section{Introduction}

COL4A1, located on chromosome 13q34, encodes the $\alpha 1$ chain of type IV collagen, a nonfibrillar protein expressed in the basement membrane of all tissues $[1,2]$. In the eyes, COL4A1 


\section{Case Reports in Ophthalmology}

is expressed in the conjunctiva, corneal endothelium and epithelium, trabecular meshwork, Schlemm's canal, lens, retinal inner limiting membrane, Bruch's membrane, and vessels [3].

Pathology resulting from the rare mutation of COL4A1 is extremely varied, with different intra- and interfamilial clinical phenotypes. Sporadic cases arise from de novo mutation and are characterized by severe clinical manifestations [4]. Age at onset is extremely variable, from fetal period to young adulthood, although cases of asymptomatic subjects or with lateonset cerebral hemorrhages have been described [5]. The phenotype variability observed in the components of families carrying the same mutation suggests a role of environmental factors in gene expression, such as head traumas or use of oral anticoagulants $[6,7]$.

Several lesions of the central nervous system related to COL4A1 mutations have been described, such as porencephaly, hydranencephaly, periventricular leukoencephalopathy, lissencephaly, schizencephaly, cerebral calcifications, intracerebral and intraventricular hemorrhages, cerebellar atrophy, intracranial aneurysms, hydrocephalus, mild ventriculomegaly, abnormal basal ganglia [4], and microencephaly [8]. COL4A1 mutation is also related to renal alterations such as renal cysts, microscopic and macroscopic hematuria, renal agenesis and atrophy, and renovascular hypertension $[9,10]$. Cardiac manifestations include Raynaud phenomenon, supraventricular arrhythmias, and mitral valve prolapse [4]. Muscular involvement has also been reported, with increased serum creatine kinase concentration [11], muscle cramps, and myopathy [4]. One case of COL4A1 mutation has been linked with severe and repeated alveolar hemorrhages [10]. Numerous ocular manifestations have been observed, such as Axenfeld-Rieger anomaly, characterized by dysgenesis of the anterior chamber angle and of the aqueous drainage structures, iris hypoplasia, iridocorneal adhesions, and corectopia. Cataract, microcornea, Peters anomaly, corneal opacities, posterior embryotoxon, glaucoma, retinal arterial tortuosity, retinal hemorrhages, optic atrophy, and anophthalmos/microphthalmos have also been reported $[4,12]$.

We report the case of a girl with microencephaly, frontal lissencephaly, parenchymal hemorrhages, almost complete corpus callosum agenesis, and hypoplasia of the optic nerve who developed a bilateral cataract at 6 months of life and in whom a novel COL4A1 mutation was identified as the genetic cause.

\section{Case Presentation}

The patient was the second daughter of nonconsanguineous Caucasian parents. Her mother was 30 years old and her father was 35 years old. There was no known exposure to any teratogens. The mother was diagnosed with pregnancy diabetes and treated with insulin. Fetal ultrasound at the 32nd gestational week showed intrauterine growth retardation without other associated findings. It was repeated at the 35th gestational week, detecting microcephaly, ventricular dysmorphism, and cerebellar hypoplasia. The child was born at 38 gestational weeks via normal spontaneous delivery, with an APGAR score of 7 and 8 at 1 and 5 min after birth, respectively. Birth weight was $2,172 \mathrm{~g}(<3 \mathrm{rd}$ percentile), length was $44.9 \mathrm{~cm}(<3 \mathrm{rd}$ percentile), and cranial circumference was $29.9 \mathrm{~cm}(<3 \mathrm{rd}$ percentile). Brain magnetic resonance imaging after 7 days showed enlargement of the periencephalic subarachnoid spaces, both under and over the tentorium, ventriculomegaly, almost complete corpus callosum agenesis, and cerebellar hemispheres and vermis hypoplasia. Porencephalic areas, delimited by hyperintense edges on T2-weighted images and hypointense edges on T1-weighted images, were observed in the cerebral parenchyma, and they were determined to be of hemorrhagic origin. Gyri defects with bilateral frontal and temporal lissencephaly were also present 


\section{Case Reports in Ophthalmology}

(Fig. 1). One month later, a computed tomography scan was performed which revealed cortical and subcortical diffuse periventricular calcifications (Fig. 2).

At birth the girl presented slight muscular hypertonia which evolved into a spastic tetraparesis in the following months. At 6 months of age the infant presented episodes of seizures which were treated with antiepileptic medical therapy, achieving good control with no relapses.

At 5 days of age, ophthalmological examination detected marked hypoplasia of the optic nerve at the fundus oculi, while no anomalies of the anterior segment were observed (Fig. 3). At 6 months of age an ophthalmological reevaluation identified a nuclear cataract (Fig. 4) that prevented evaluation of the fundus oculi. The child underwent bilateral cataract extraction, without intraocular lens implantation, and anterior vitrectomy with capsulotomy by vitrectome, with an uncomplicated postoperative course. One week after surgery she developed increased intraocular pressure in the left eye, resistant to medical therapy, and 1 month later the right eye developed a pupillary membrane. Transconjunctival laser cyclophotocoagulation was performed on $360^{\circ}$ in the left eye and the pupillary membrane was removed in the right eye. Subsequently, intraocular pressure in the left eye normalized with topical therapy and the optical axis of the right eye was free. Despite optical correction with positive lenses, visual acuity remained uncertain light perception in both eyes after the second surgery.

Considering the patient's clinical manifestations, sequencing of the COL4A1 gene was performed. The test results were positive for the heterozygous mutation c.2716+2T $>C$, which affects a consensus sequence for splicing. Both mother and father were negative for mutation.

\section{Discussion}

COL4A1 encodes the proa1 (IV) chain, which with the proa 2 (IV) chain, encoded by the COL4A2 gene, leads to type IV collagen production. The two chains are assembled as hetero-

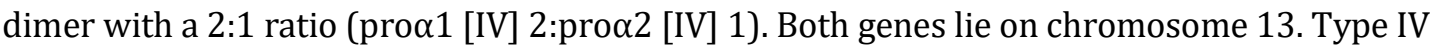
collagen is a nonfibrillar collagen and is the main component of the basement membrane of many tissues [4]. It consists of three major domains: the amino terminal 7S domain, which intervenes in intermolecular crosslinking and macromolecular organization, the central triple helix, and a carboxy-terminal domain, noncollagenous, which is responsible for the initiation of heterodimer assembly [13]. Mutations of COL4A1 are extremely rare and cause alteration of the basement membrane in many organs, including the eye.

Typical central nervous system manifestations of COL4A1 mutations are microcephaly, porencephaly, lissencephaly, and dilatation of cerebral ventricles, and are also present in this novel mutation. Brain magnetic resonance imaging, performed on the 7 th day of age, showed alterations compatible with brain hemorrhages. These likely occurred during intrauterine life, in accordance with previous reports on intracerebral hemorrhages before birth [1, 14], while late-onset brain hemorrhages are often associated with trauma [7]. In fact, the disease presents different clinical manifestations and age at onset, based on the type of mutation and environmental factors that influence the phenotype.

This novel mutation is accompanied by two different entities and previously undescribed in association with the known mutations of COL4A1: almost complete agenesis of the corpus callosum and optic nerve hypoplasia. Type IV collagen plays a role in embryogenesis, angiogenesis, cellular migration, and differentiation [15], which could explain the onset of the aforementioned alterations. Spastic tetraparesis and epileptic crises could be attributed to the 
numerous cerebral parenchymal hemorrhages, a manifestation often related to COL4A1 mutations.

The mechanism of cataract formation is not precisely determined, although a hypothesis related to oxidative stress has been proposed. Unfolded type IV collagen chains accumulate in rough endoplasmic reticulum whenever COL4A1 mutation c. $2345 \mathrm{G}>\mathrm{C}$ is present. Progressive accumulation in rough endoplasmic reticulum causes oxidative stress and, if not opposed by other mechanisms, such as reduction of protein synthesis or increased clearance of unfolded protein, causes cell apoptosis, eventually leading to cataract development [13].

The patient also developed glaucoma in the left eye. The timing of the elevation of intraocular pressure seems unlikely to be related to cataract surgery, although rare cases of early pediatric glaucoma after cataract surgery have been described. Our hypothesis is that the pathological basis of this case of glaucoma could have been an undocumented alteration to angle structures, frequently described in association with COL4A1 mutations. In addition, the child developed a fibrotic pupillary membrane after cataract extraction, a condition already described in other patients with COL4A1 mutation [12].

In conclusion, we report a case of optic nerve hypoplasia and almost complete corpus callosum agenesis in a patient with disorders related to a novel mutation in COL4A1. An extensive research of the scientific literature did not find other cases with this mutation. It is recommended to plan a follow-up, due to the unpredictable timing of onset of complications which are not always present at birth and could threaten the patient's vision if left unchecked.

\section{Statement of Ethics}

This work was performed according to the guidelines for human studies and was performed ethically in accordance with the World Medical Association Declaration of Helsinki. The patient's legal tutor gave informed consent to publish the case, including images. Information revealing the subject's identity was avoided. The patient is not identified by her real name.

\section{Disclosure Statement}

L. Grego, S. Pignatto, N. Rassu, E. Passone, P. Cogo: no conflicts of interest. P. Lanzetta: Bayer, Centervue, Novartis Pharma AG.

\section{Funding Sources}

None.

\section{Author Contributions}

Dr. L. Grego took the lead in writing the manuscript. Dr. N. Rassu provided imaging and language support. All authors discussed the results and contributed to the final manuscript. 


\section{Case Reports in Ophthalmology}

\section{References}

1 Durrani-Kolarik S, Manickam K, Chen B. COL4A1 Mutation in a Neonate With Intrauterine Stroke and Anterior Segment Dysgenesis. Pediatr Neurol. 2017 Jan;66:100-3.

2 Vahedi K, Alamowitch S. Clinical spectrum of type IV collagen (COL4A1) mutations: a novel genetic multisystem disease. Curr Opin Neurol. 2011 Feb;24(1):63-8.

3 Rødahl E, Knappskog PM, Majewski J, Johansson S, Telstad W, Kråkenes J, et al. Variants of anterior segment dysgenesis and cerebral involvement in a large family with a novel COL4A1 mutation. Am J Ophthalmol. 2013 May;155(5):946-53.

4 Meuwissen ME, Halley DJ, Smit LS, Lequin MH, Cobben JM, de Coo R, et al. The expanding phenotype of COL4A1 and COL4A2 mutations: clinical data on 13 newly identified families and a review of the literature. Genet Med. 2015 Nov;17(11):843-53.

5 Magnin E, Ayrignac X, Berger E, Mine M, Tournier-Lasserve E, Labauge P. Late diagnosis of COL4A1 mutation and problematic vascular risk factor management. Eur Neurol. 2014;72(3-4):150-2.

6 Lemmens R, Maugeri A, Niessen HW, Goris A, Tousseyn T, Demaerel P, et al. Novel COL4A1 mutations cause cerebral small vessel disease by haploinsufficiency. Hum Mol Genet. 2013 Jan;22(2):391-7.

7 Plancher JM, Hufnagel RB, Vagal A, Peariso K, Saal HM, Broderick JP. Case of Small Vessel Disease Associated with COL4A1 Mutations following Trauma. Case Rep Neurol. 2015 Jun;7(2):142-7.

8 Shah S, Ellard S, Kneen R, Lim M, Osborne N, Rankin J, et al. Childhood presentation of COL4A1 mutations. Dev Med Child Neurol. 2012 Jun;54(6):569-74.

9 Kuo DS, Labelle-Dumais C, Gould DB. COL4A1 and COL4A2 mutations and disease: insights into pathogenic mechanisms and potential therapeutic targets. Hum Mol Genet. 2012 Oct;21(R1):R97-110.

10 Abe Y, Matsuduka A, Okanari K, Miyahara H, Kato M, Miyatake S, et al. A severe pulmonary complication in a patient with COL4A1-related disorder: A case report. Eur J Med Genet. 2017 Mar;60(3):169-71.

11 Tonduti D, Pichiecchio A, La Piana R, Livingston JH, Doherty DA, Majumdar A, et al. COL4A1-related disease: raised creatine kinase and cerebral calcification as useful pointers. Neuropediatrics. 2012 Oct;43(5):283-8.

12 Deml B, Reis LM, Maheshwari M, Griffis C, Bick D, Semina EV. Whole exome analysis identifies dominant COL4A1 mutations in patients with complex ocular phenotypes involving microphthalmia. Clin Genet. 2014 Nov;86(5):475-81.

13 Xia XY, Li N, Cao X, Wu QY, Li TF, Zhang C, et al. A novel COL4A1 gene mutation results in autosomal dominant non-syndromic congenital cataract in a Chinese family. BMC Med Genet. 2014 Aug:15(1):97.

14 Colin E, Sentilhes L, Sarfati A, Mine M, Guichet A, Ploton C, et al. Fetal intracerebral hemorrhage and cataract: think COL4A1. J Perinatol. 2014 Jan;34(1):75-7.

15 Chen Z, Migeon T, Verpont MC, Zaidan M, Sado Y, Kerjaschki D, et al. HANAC Syndrome Col4a1 Mutation Causes Neonate Glomerular Hyperpermeability and Adult Glomerulocystic Kidney Disease. J Am Soc Nephrol. 2016 Apr;27(4):1042-54. 


\section{Case Reports in Ophthalmology}

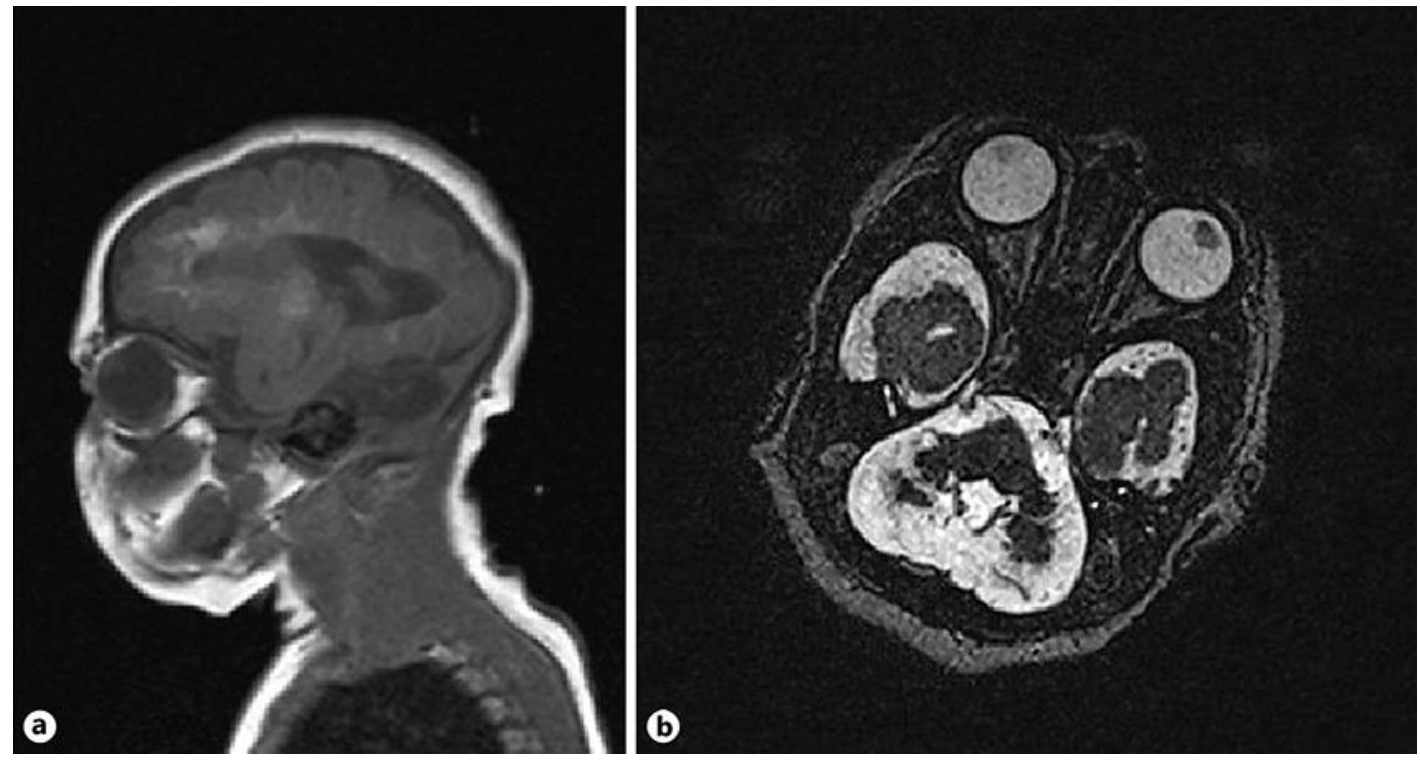

Fig. 1. a, b Brain magnetic resonance imaging showing optic nerve hypoplasia and almost complete corpus callosum.

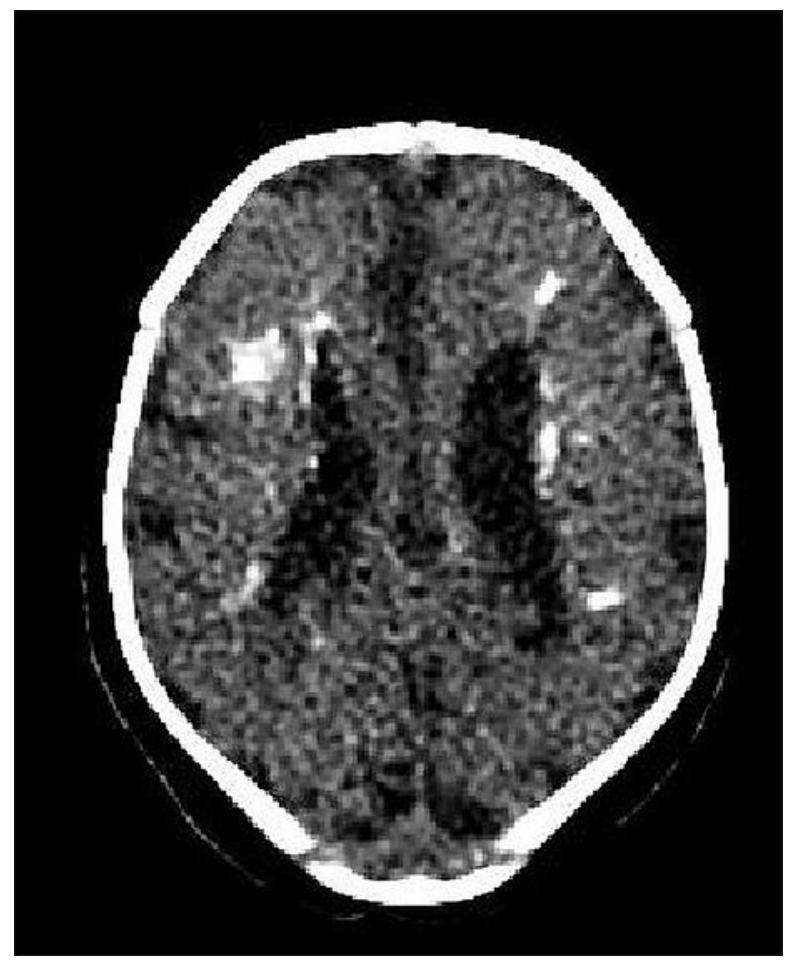

Fig. 2. Computed tomography scan showing subcortical diffuse periventricular calcifications. 

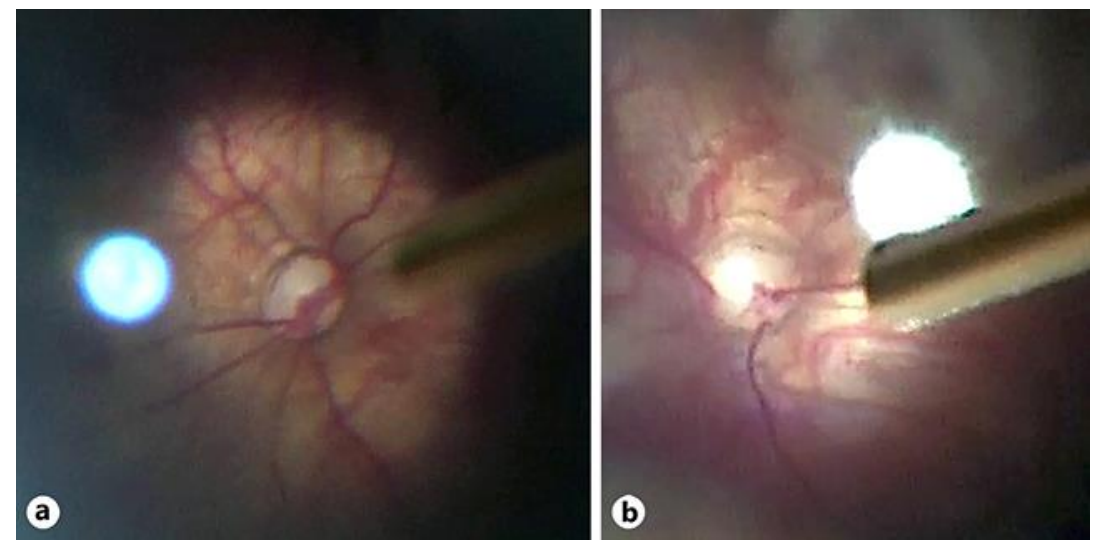

Fig. 3. a, b Hypoplasia of the optic nerve head.
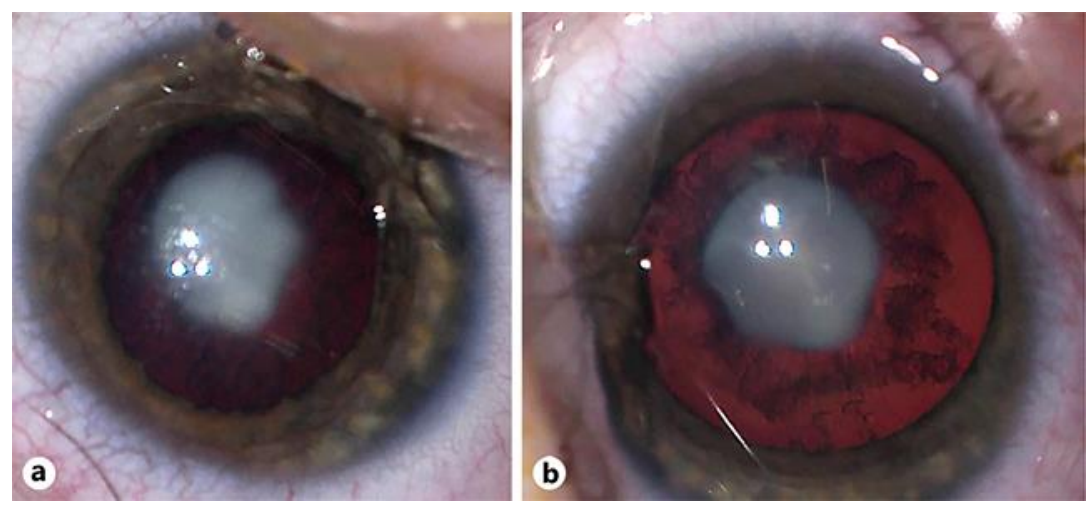

Fig. 4. a, b Bilateral nuclear cataract before surgery. 